\title{
Intelligent Modulation Recognition System and its Implementation using MATLAB
}

\author{
Prof. Arun Pawar, Mr. Dharmesh Dhabliya \\ Professor, Department of Mechanical Engineering, Bharti Vidyapeth Pune India \\ Dean, Research and Development, Yashika Publications India \\ dharmesh@yashikapublications.com
}

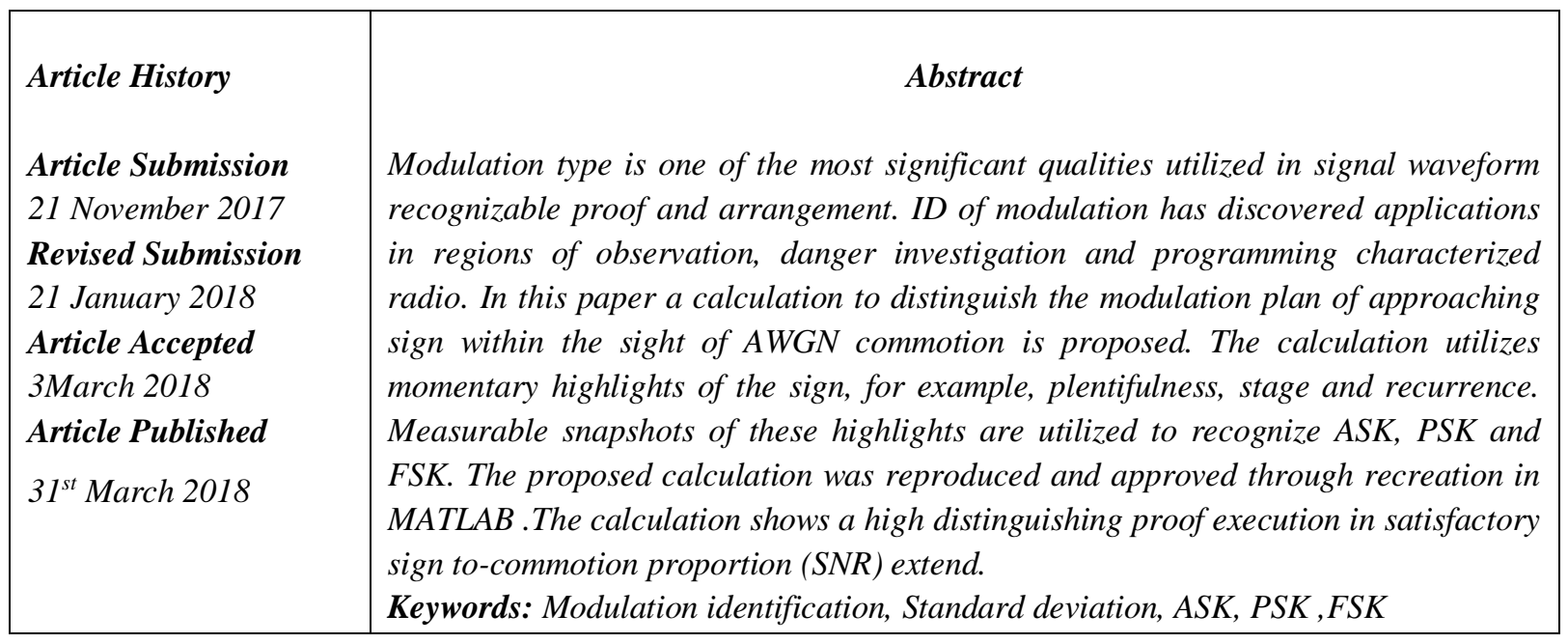

\section{Introduction}

Inventions in radio networks have got conceivable change in the recipient arrangement as indicated by the shifting correspondence norms. Versatile demodulation of the approaching sign or visually impaired demodulation for example demodulation of the sign with no earlier data is a functioning region in examine. Distinguishing proof the modulation plan of the got signal is consequently the key in this situation. There are numerous different applications where modulation distinguishing proof assumes a crucial job. This incorporates military applications, for example, reconnaissance, danger investigation and non military personnel applications, for example, impedance recognizable proof and range the board [1][4].

Before, much work has been directed on modulation distinguishing proof. The recognizable proof procedures, which had been utilized to separate the sign highlights vital for computerized modulation acknowledgment, incorporate phantom based list of capabilities, higher request cumulates (HOC), star grouping shape, and wavelets changes.

Modulation classifiers are commonly isolated into two classifications. The main classification depends on choice hypothetical methodology while the second on design acknowledgment. The choice hypothetical methodology or Maximum Likelihood Approach [6][7] is a probabilistic arrangement dependent on from the earlier information on likelihood capacities and certain speculations. Then again, the example acknowledgment approach [2][3] depends on separating some essential qualities of the sign called highlights. This methodology is commonly isolated into two subsystems: the highlights extraction subsystem and the classifier subsystem.

\section{Related Works}

Asoke K. Nandi[2] presented calculations for simple and computerized modulations acknowledgment. The calculation uses the choice hypothetical methodology wherein a lot of choice rules for distinguishing various sorts of modulations were utilized. In the choice hypothetical calculation it is discovered that the general 
achievement rate was over $94 \%$ at the sign to-clamor proportion (SNR) of $15 \mathrm{~dB}$, while in the ANN calculation the general achievement rate is over $96 \%$ at the SNR of $15 \mathrm{~dB}$ [5][8].

Durga Digdarsini,[9] utilized a wavelet change based calculation which includes multi-rate signal preparing, was acknowledged to recognize QAM, PSK and FSK computerized modulation signal in loud condition. The methodology was to utilize wavelet change to separate the transient attributes in a computerized modulation sign to distinguish the sort of modulation

The example acknowledgment approach is progressively strong and simpler to execute if the best possible highlights set is picked. It requires no from the earlier data of the got signal, But the exhibition was seen as profoundly subject to motion toward commotion proportion [10].

\section{Proposed Modulation Identification Methodology}

In this paper a calculation to recognize the tweak of the got signal went to an added substance white Gaussian channel (AWGN) is created. This calculation is actualized by confirming if the blocked sign contains data in momentary sufficiency, prompt stage or immediate recurrence. Second request measurable second for example standard deviation of these immediate highlights fills in as the choice models to order the got signal.

Consider that the sign is blocked for a time span of $\mathrm{K}$ seconds. The sign is inspected at pace of $\mathrm{fs} \mathrm{Hz}$. The examining recurrence ought to be multiple times more noteworthy than most extreme recurrence in the sign range. The examples are then gathered $N s$ tests per fragment bringing about $M$ portions ( $M=K$ fs $/ N s)$. Sort of adjustment is distinguished for every single fragment.

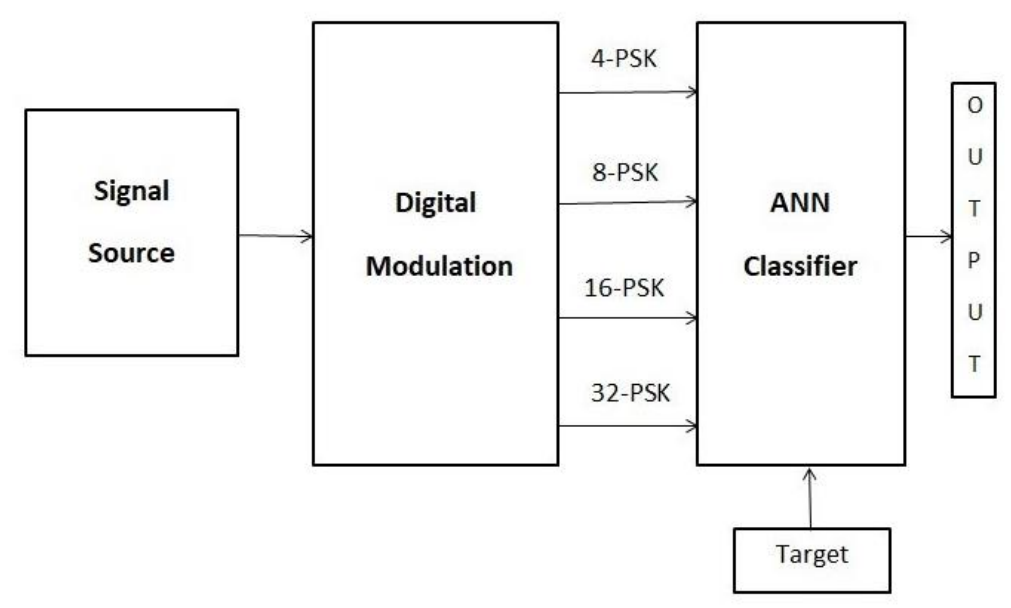

Fig 1: Functional block of Modulation Identification System

First the prompt highlights of the sign should be determined. This is finished by performing Hilbert change on the got tests. The Hilbert change is utilized to determine the scientific portrayal of a sign $u(t)$. This implies genuine sign $u(t)$ is stretched out into complex structure that is gotten from the expository portrayal. For each section, in this manner the momentary amplitudes $\{\mathrm{A}(\mathrm{i}): \mathrm{I}=1,2 . . \mathrm{NS}$,$\} and quick stages \{\varnothing(\mathrm{i}): \mathrm{I}=1,2, ., \mathrm{Ns}$,$\} are$ then extricated, where the file "I" speaks to that a(i) and $\varnothing$ (I) are for the $\mathrm{i}^{\text {th }}$ complex example. A basic plan is utilized to open up the quick stages, bringing about an opened up stage grouping $\{\varnothing \mathrm{UW} \mathrm{W}(\mathrm{i}): \mathrm{I}=1,2, \ldots, \mathrm{Ns}\}$. The opening up method is as clarified beneath. $\mathrm{Ck}$ is the amendment applied to the stage to open up.

The unwrapped phase is obtained by

$$
\emptyset \mathrm{UW}(\mathrm{i})=\varnothing(\mathrm{i})+\mathrm{Ck}(\mathrm{i})
$$


Phase opening up makes the prompt phase a persistent capacity of time. From the opened up phase there is a need to recognize the non direct phase of the message signal. This is gotten by expelling the straight phase part. The non direct phase shows the message. The nonlinear phases $\{\varnothing N L(i): I=1,2 \ldots$, Ns, $\}$ are then acquired by evacuating the straight phase inferable from the transporter recurrence from the opened up phases.

$$
\emptyset \mathrm{NL}(\mathrm{i})=\varnothing \mathrm{UW}(\mathrm{i})-(2 \pi \mathrm{fc} / \mathrm{fs}) * \mathrm{i}
$$

Based on the above sequences, some other sequences are defined as follows:

Normalized instantaneous amplitude can be obtained as

$$
\operatorname{an}(\mathrm{i})=\mathrm{a}(\mathrm{i}) /\left(\frac{1}{\mathrm{~N}_{\mathrm{s}}} \sum_{\mathrm{i}=1}^{\mathrm{Ns}} \mathrm{a}(\mathrm{i})\right)
$$

The centralized normalized instantaneous amplitude is given by

$$
\operatorname{acn}(\mathrm{i})=\operatorname{an}(\mathrm{i})-1
$$

The functional flow chart of the modulation classification is depicted in the figure 1. It indicates the method in which the features need to be extracted so that the incoming signals can be classified accordingly. For each feature a threshold is fixed and depending on the value of the received signal it is grouped into two categories, each category is further tested for another feature.

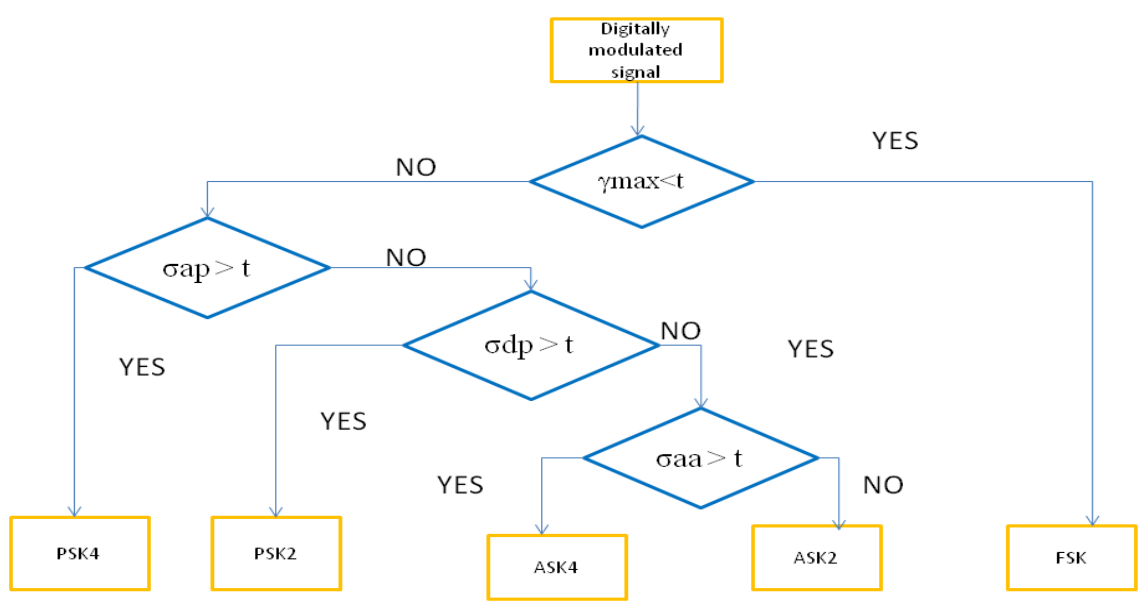

Fig 2: Functional flowchart for automatic identification of digital modulations.

\section{Simulation Results}

The simulations presented in this paper were simulated in MATLAB 2013. The parameters used in the simulation is shown in the table 1 . The algorithm was verified and validated for various modulation schemes ASK, PSK and FSK. The signals were passed through AWGN channel with varying signal to noise ratio. The instantaneous features were extracted and statistical moments were calculated.

Table 1: Simulation Parameters

\begin{tabular}{|c|c|}
\hline Carrier Frequency & $150 \mathrm{KHz}$ \\
\hline Sampling Frequency & $32 \mathrm{MHz}$ \\
\hline Bit rate & $10 \mathrm{KHz}$ \\
\hline No of segments & 100 \\
\hline Signal Intercepted & $20 \mathrm{~ms}$ \\
\hline
\end{tabular}


The simulated result for $\gamma \max$ is shown in the figure 3. The value of $\gamma \max$ is almost zero for FSK modulation scheme. This is shown in figure 4 .

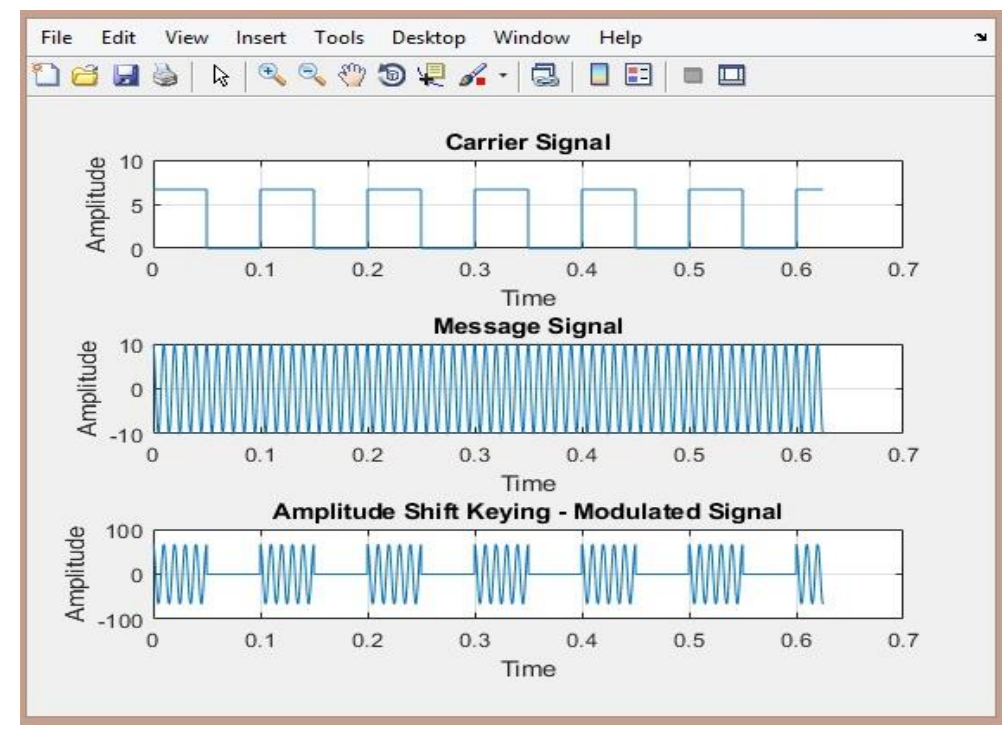

Fig 3 : Simulation of Amplitude Shift Keying Frequency Shift Keying

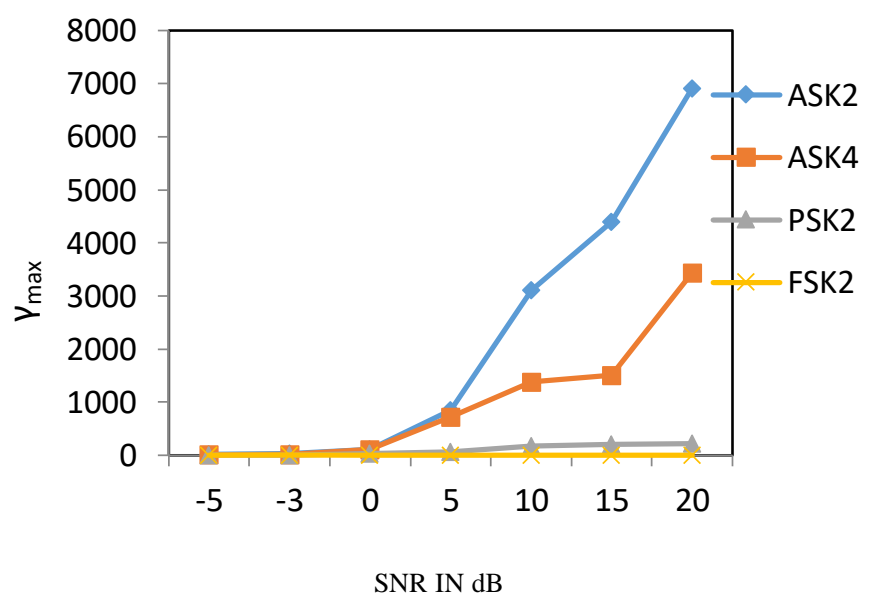

Fig 4 : Gamma_max Vs SNR

The simulated values of $\sigma$ a validates that it can be used to separate ASK4 and ASK2. The simulated graph is shown in the figure 5 .

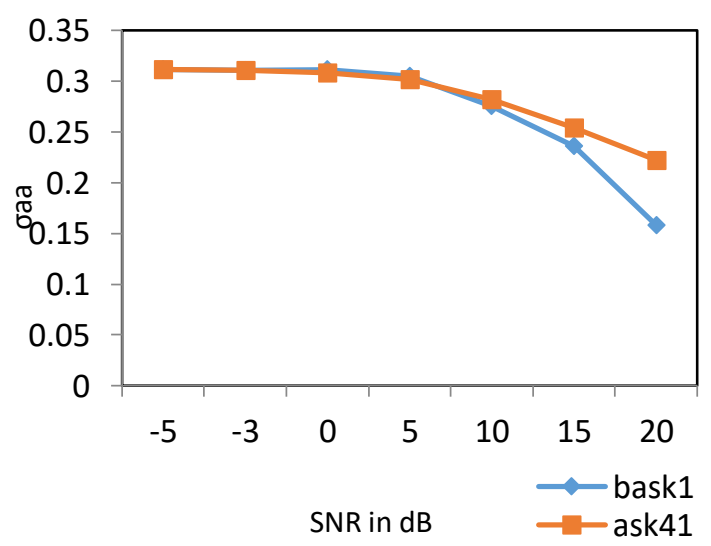

Fig 5:Sigma_aa Vs SNR 
The oap and $\sigma \mathrm{dp}$ explained before is used to identify if the received signal has any phase information. The graph is as plotted in the figure 6 and 7.

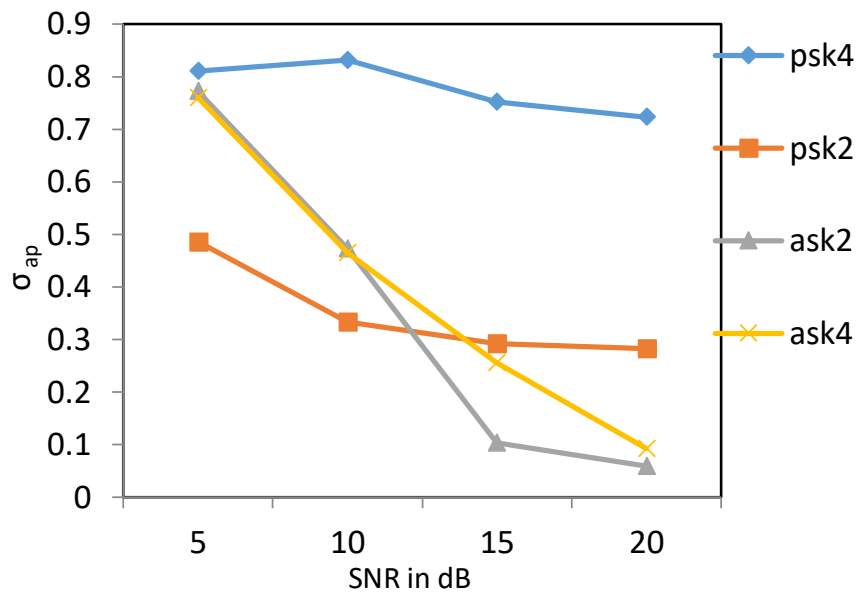

Fig 6: Sigma_apVs SNR

The $\sigma_{a p}$ value plotted is graphs shows that PSK4 has higher standard deviation then other modulation schemes. This is because the ASK2 and ASK4 has no absolute phase information, centralized normalized phase for PSK2 is \pm 1 (in the absence of noise ) and hence taking absolute value the standard deviation of this will result in a small value.

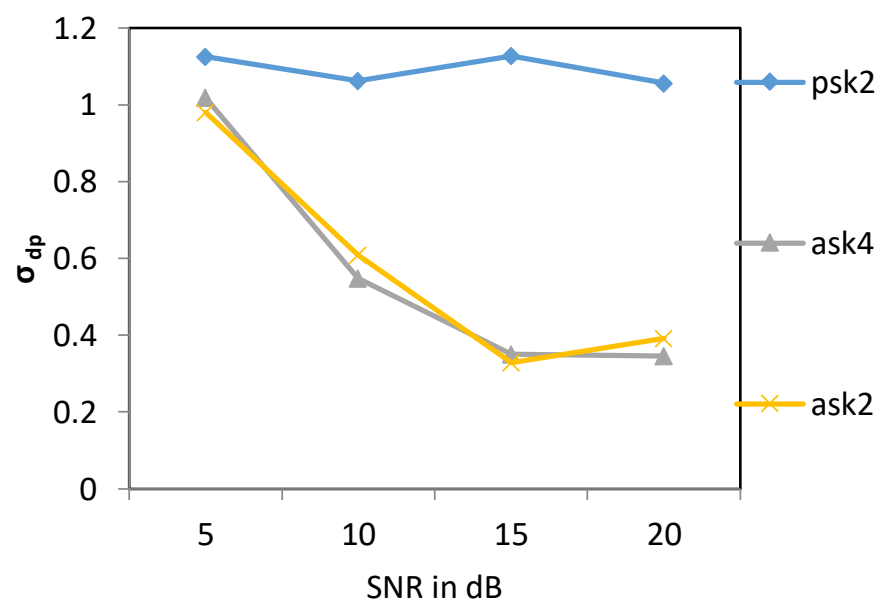

Fig 7:Sigma_dp Vs SNR

The $\sigma_{\mathrm{dp}}$ takes the direct phase information. The centralized normalized phase of ASK2 and ASK4 is zero (in ideal scenario), and hence the standard deviation will be a very low value as compared to PSK2. It very well may be seen from results that the proposed highlights can be utilized to recognize the regulation plan of the got signal even in nearness of AWGN clamor.

The criteria proposed will be able to differentiate binary and quadrature modulation schemes. In order to separate higher order modulation schemes there is need to go for higher order moments. The proposed features are meaningful and easy to implement. The simulation results are shown in figure 8 and figure 9 respectively. 


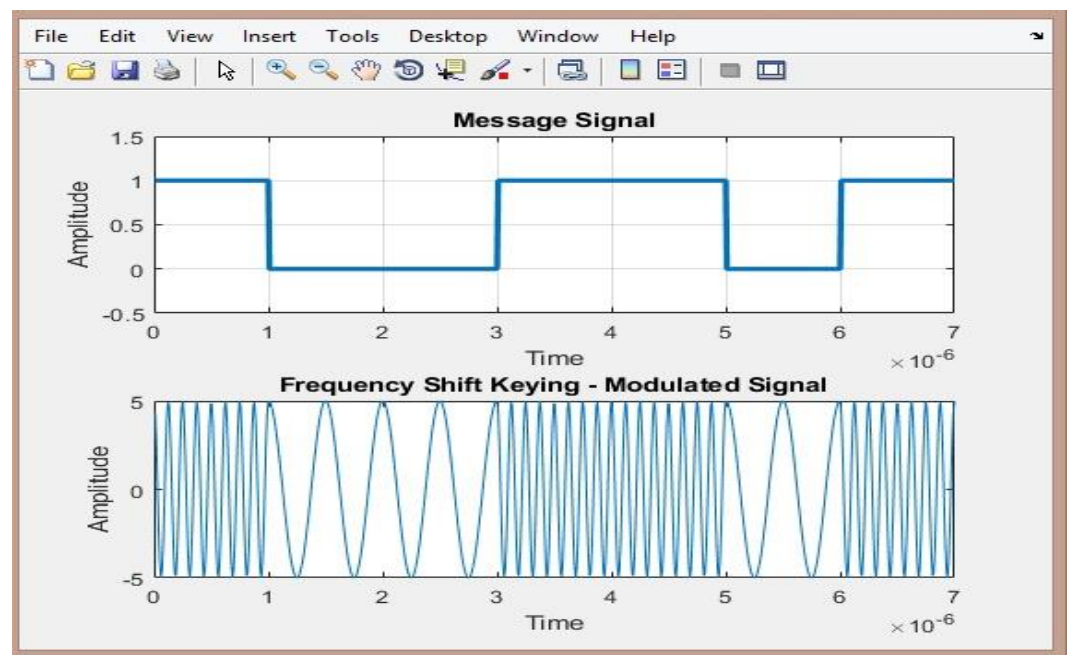

Fig 8: Simulation waveform of Frequency Shift Keying

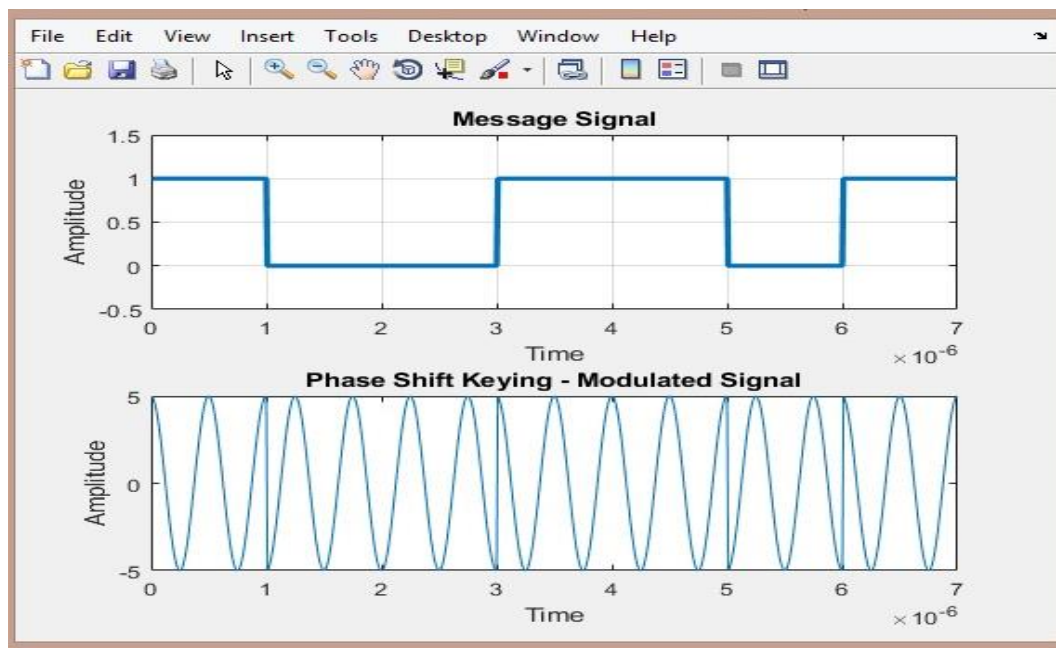

Fig 9: Simulation of Phase Shift Keying and Binary Phase Shift Keying

The component separation between two distinct gatherings of regulation kinds by and large declines as the SNR diminishes. This implies the choice edge must be chosen in a smaller range if the SNR is lower. The exhibition of the standards is better if the signal to noise proportion is more noteworthy than 10dB. This makes the calculations just ready to work for higher SNR. The comparison is shown in figure 10.

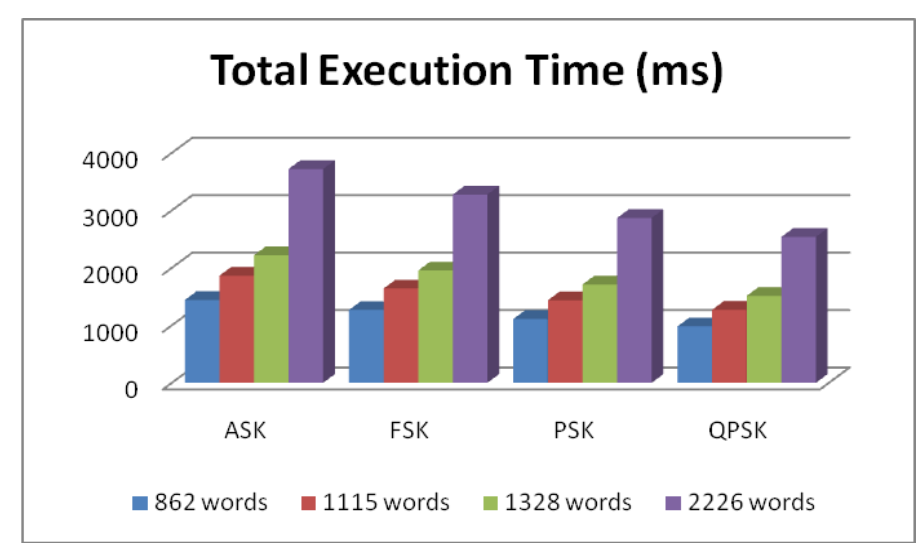

Fig 10: Comparison chart of various modulation execution time 


\section{Conclusion}

The calculation for an Automatic modulation acknowledgment framework was proposed in this paper. The calculation targets distinguishing advanced modulation plans, for example, PSK, FSK and ASK. The proposed calculation utilizes a component extraction dependent on the quick abundance, recurrence and stage. The calculation is basic and simple to execute. The exhibition is better if the SNR is more noteworthy than 10dB. The downside of the proposed plot is that its presentation debases with lower SNR. Further research should be possible to ad lib the highlights to be commotion free. The recipient executed utilizing this calculation will have the capacities to change the total setups as per the correspondence principles and adaptively demodulate the approaching sign. This will be exceptionally helpful in fourth era correspondence framework where a beneficiary can get more than one kind of modulation with single recipient.

\section{References}

[1] K. Hassan, Dayoub, W.Hamouda ,and M .Berbineau, "Automatic Modulation Recognition Using Wavelet Transform and Neural Networks in Wireless Systems" EURASIP Journal on Advances in Signal Processing Volume 2010

[2] H. S. Khallaf, H. M. H. Shalaby, J. M. Garrido-Balsells and S. Sampei, "Performance analysis of a hybrid QAM-MPPM technique over turbulence-free and gamma-gamma free-space optical channels," in IEEE/OSA Journal of Optical Communications and Networking, vol. 9, no. 2, pp. 161-171, Feb. 2017, doi: 10.1364/JOCN.9.000161.

[3] Asoke K. Nandi," Algorithms for Automatic Modulation Recognition of Communication Signals" IEEE Transactions On Communications, Vol. 46, No. 4, April 1998

[4] Durga Digdarsini, Mahesh Kumar, Gopichand Khot, T.V.S. Ram, V.K.Tank" FPGA Implementation of Automatic Modulation Recognition System for Advanced SATCOM System”, 2014 International Conference on Signal Processing and Integrated Networks (SPIN)

[5] P. Prakasam and M. Madheswaran ,"Digital Modulation Identification Model Using Wavelet Transform and Statistical Parameters" Hindawi Publishing Corporation ,Journal of Computer Systems, Networks, and Communications Volume 2008,

[6] C. I. Siyad and V. Neethu, "Performance analysis of diversity techniques for OFDM system using trellis coded OAM-QAM union modulation," 2017 International Conference on Intelligent Computing and Control (I2C2), Coimbatore, 2017, pp. 1-5, doi: 10.1109/I2C2.2017.8321842.

[7] A. Swami and B.M . Sadler, "Hierarchical digital modulation classification using cumulants," IEEE Transact ions on Communications, vol. 48, no. 3, pp. 416-429, 2000

[8] Asoke K. Nandi, E.E.Azzouz "Automatic identification of digital modulation types", Signal processing, 28 November1994

[9] Qinghua Shi,Y.Karasawa "Automatic Modulation Identification Based on the Probability Density Function of Signal Phase", IEEE transactions on communications, Vol. 60, No. 4, April 2012

[10] C. T. Manimegalai, S. Gauni, N. Raghavan and T. R. Rao, "Investigations on suitable modulation techniques for visible light communications," 2017 International Conference on Wireless Communications, Signal Processing and Networking (WiSPNET), Chennai, 2017, pp. 1818-1822, doi: 10.1109/WiSPNET.2017.8300075. 